\title{
The Mosaics of the Church of Santa Maria Do Freixo (Marco De Canaveses): Reflections on Its Meaning in the Context of the Late Musivaria of the Douro Valley \\ Santa Maria Do Freixo Kilise Mozaikleri (Marco De Canaveses): Douro Vadisi Geç Musivaria Kontekstinin Anlamı Üzerine Düşünceler
}

(Received 12 February 2017, accepted after revision 25 May 2017)

\begin{abstract}
In this paper, we present the polychromatic roman mosaics with a geometric organization and a vegetalist decoration discovered during the archaeological excavation that took place in 2001 in Santa Maria do Freixo church (Northern Portugal). After describing the ruins and the mosaics, a brief contextualization (including the formal analysis of the building and of the mosaics and its local and regional geographic context) is given in order to try to understand its social and cultural meanings.
\end{abstract}

Keywords: Polychromatic, geometric, vegetalist, Douro Valley, Late Antiquity.

\section{$\ddot{\mathrm{O} z}$}

Bu çalışmada, 2001 yılında Santa Maria do Freixo Kilisesi'nde (Kuzey Portekiz) gerçekleştirilen arkeolojik kazı sırasında keşfedilen geometrik ve bitkisel süslemeye sahip polikrom Roma mozaikleri sunulmuştur. Arkeolojik kalıntıları ve mozaikleri tanımladıktan sonra, klsa bir kontekst değerlendirmesi(binanın ve mozaiklerin analizi ile yerel ve bölgesel coğrafi kontekst de dahil olmak üzere) yapılacak, sosyal ve kültürel manaları anlaşılmaya çalışılacaktır.

Anahtar Kelimeler: Polikrom, geometrik, bitkisel, Douro Vadisi, Geç Antik.

Les mosaïques ici présentées ont été identifiées pendant l'intervention archéologique réalisée dans le sous-sol de l'église de Santa Maria do Freixo (clientèle de Marco, commune de Marco de Canaveses, district de Porto, Nord du Portugal). Il s'agit d'un site archéologique, sous la gestion de la Direção Regional de Cultura do Norte, situé dans le bassin hydrographique du fleuve Douro et dans la vallée d'un de ses affluents, le Tâmega (Fig. 1).

Le local correspond à l'acropole de Tongobriga, nom prélatin d'un bourg fortifié qui a connu un fort développement urbain après la fin du $1^{\text {er }}$ siècle après J.-C. comme conséquence de sa promotion à capital de civitas (Dias 1997).

\footnotetext{
* António Manuel de Carvalho Lima, Archéologue, Estação Arqueológica do Freixo/Direção Regional de Cultura do Norte. E-mail: amlima@culturanorte.pt

Nous remercions à Jorge M. S. Martins Araújo pour la révision et traduction du texte et à António Freitas l'élaboration de plans et chartes.
} 


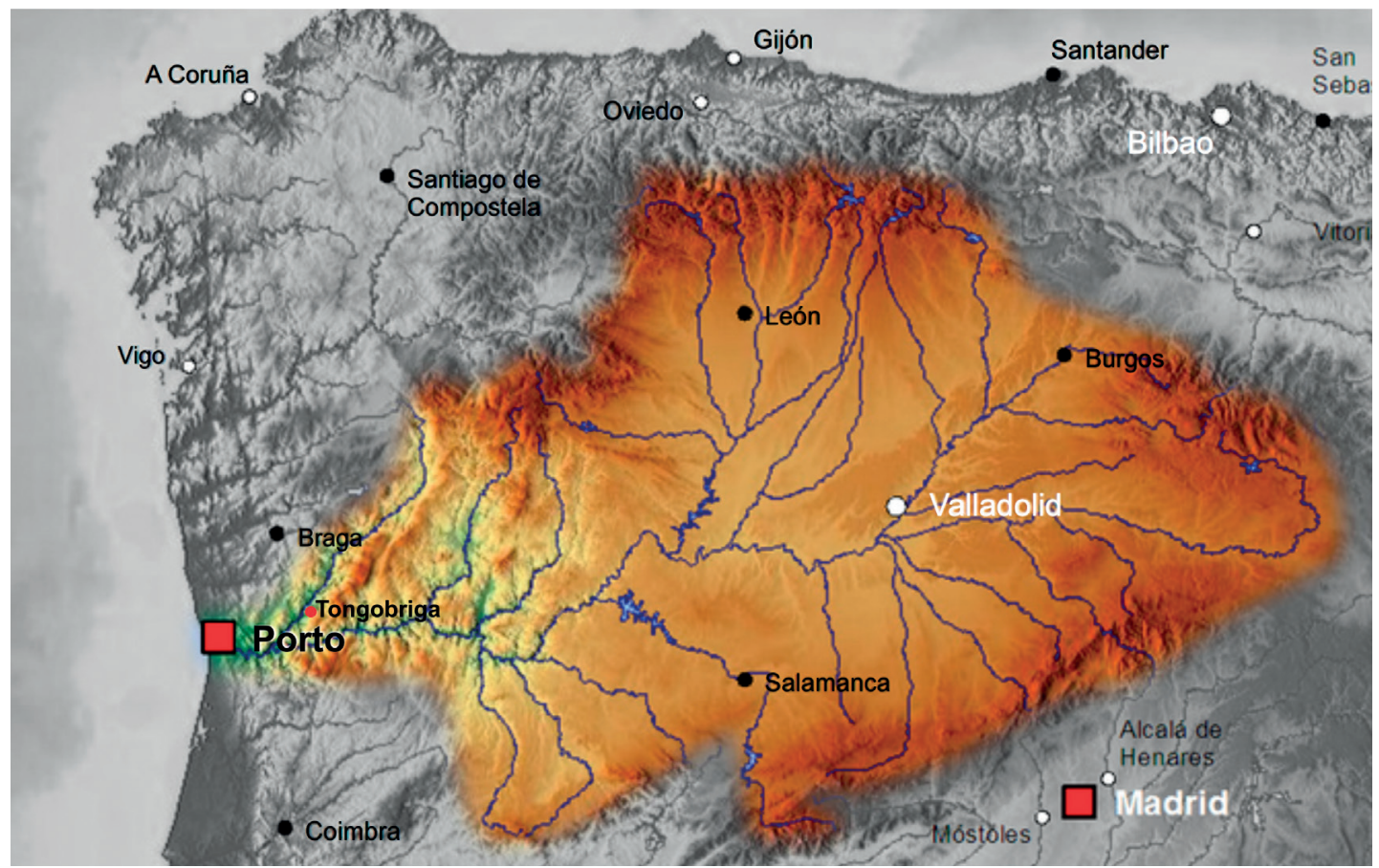

\section{Le plan du bâtiment pavé de mosaïque}

Il s'agit d'un long bâtiment - beaucoup plus grand que tous ceux qui ont déjà été exhumés dans le périmètre muraillé de la Tongobriga romaine - qui aurait au moins trois compartiments, pavés en mosaïque, communiquant entre eux et aligné E-W (Fig. 2, A, B - C).

Deux de ces espaces (Fig. 2, espaces A et C) formeraient un rectangle avec la longueur totale de $24,2 \mathrm{~m}=110$ palmi $(11$ mètres $=50$ palmi dans l'espace $\mathrm{A}$ et 13,2 mètres $=60$ palmi dans l'espace $\mathrm{C}$ ) par $8,14 \mathrm{~m}$ ( 7 palmi) de largeur.

À l'ouest, il y a aussi un troisième espace pavé de mosaïque (Fig. 2, espace B). Malheureusement, la petite zone qui a été fouillée a été intensivement utilisée
Figure 1

Localisation de Tongobriga (Santa Maria do Freixo) dans le nord de la Péninsule Ibérique et Vallée du Douro

Figure 2

Bâtiment pavé de mosaïques sous l'actuelle église de Santa Maria do Freixo. Compartiments (A, B, C), éléments architectoniques $(\mathrm{a}, \mathrm{b}, \mathrm{c})$, et accès / axes de circulation (1à 5)

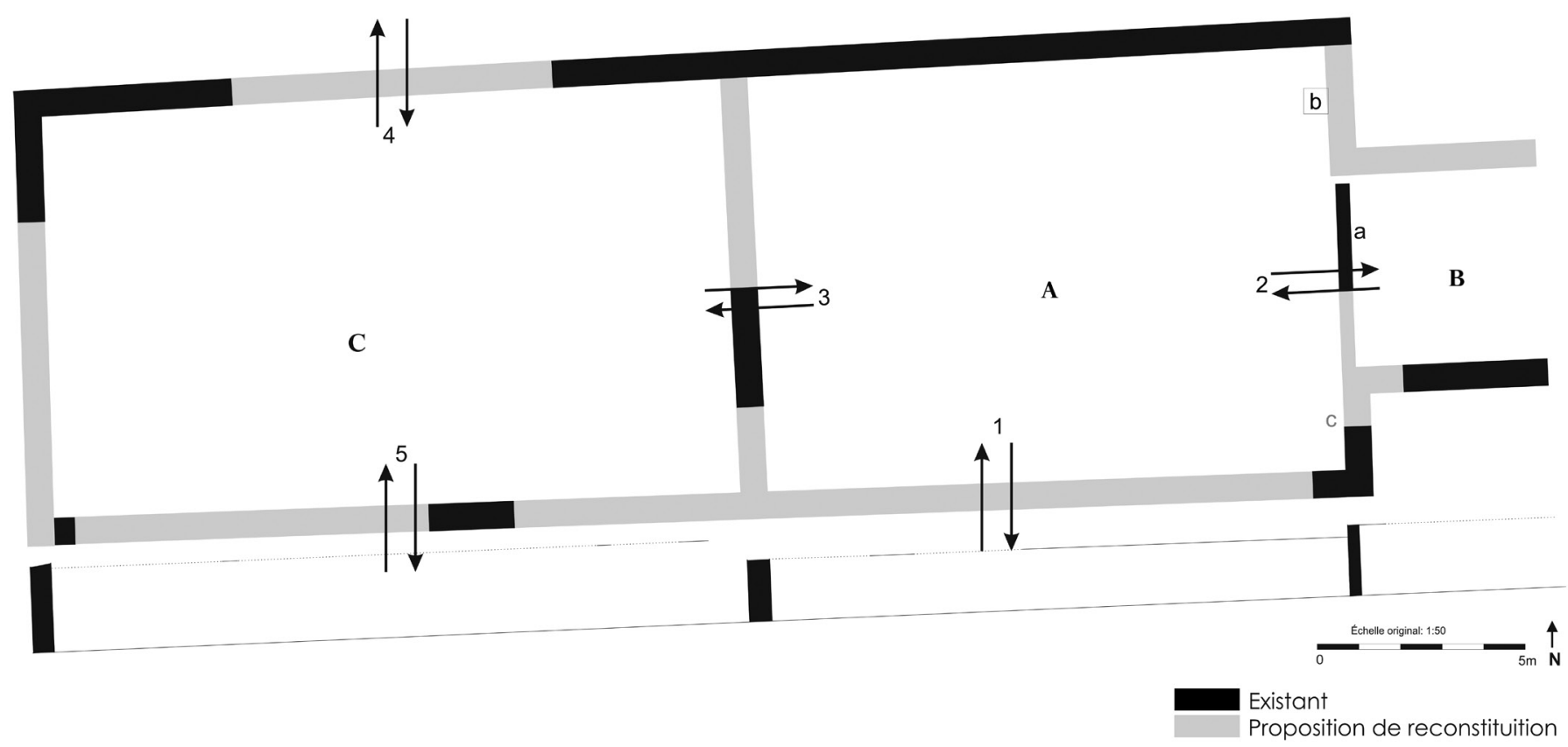


comme un espace de cimetière, et l'espace de l'autel principal n'était pas sujet à intervention. Nous avons ici sa limite ouest, qui a révélé une remarquable continuité parce qu'elle correspond, encore aujourd'hui, à l'arc triomphal qui marque le passage entre la nef et le chœur de l'église. De ce passage, nous avons encore du seuil original et une grande partie de la base de la paroi où le même était implanté (Fig. 2, a). Nous ne savions pas sa configuration, interne et externe, ainsi que sa profondeur totale. On peut estimer la largeur intérieure minimale à environ 4,5 mètres, ce qui, ajouté à l'épaisseur de ses murs $(0,5 \mathrm{~m}+0,5 \mathrm{~m})$, fait la moitié de la largeur de l'espace A. En ce qui concerne sa profondeur, cela ne pourrait être jamais moins de 3,9 mètres, attendant à la limite du mur détecté sous la paroi sud du sanctuaire.

\section{L'organisation de l'espace intérieur du bâtiment : secteurs fonctionnels et axes de circulation}

En absence d'autres éléments architectoniques qui nous permettent d'évaluer cette organisation de son espace intérieur, il est remarquable la présence, in situ, d'une pierre de taille quadrangulaire, avec 2 palmi $(=44 \mathrm{~cm})$ de largeur qui a servi comme stylobate pour une colonne ou pilastre, proche de l'angle NE de la nef de l'église actuelle (Fig. 2, b), un signe de que le même espace intérieur pourrait être rythmé par une groupe de colonnes dont nous ne pouvons pas déterminer le numéro.

Ce qui est certain c'est que cette base est appuyée à la paroi sur laquelle pose l'arc triomphal actuel et sur laquelle s'appuie le pavement de mosaïque, ce qui ne laisse aucun doute quant à l'utilisation simultanée des deux pavements dans un cadre d'un même programme architectonique.

En ce qui concerne les axes de circulation, rien n'indique que ce bâtiment avait une entrée sur sa façade occidentale, par contraste avec le temple actuel.

Par l'espace qui est maintenant connu comme Largo do Cruzeiro ont auraient accès (Fig. 2, ouverture 1) à l'axe central d'un compartiment (espace A). D'ici, on pouvait avoir accès à l'espace B (Fig. 2, B), situé à l'Est, par un passage perpétué, au fil de toutes ces siècles, par l'arc triomphal de l'église (Fig. 2, ouverture 2). L'accès à l'espace $\mathrm{B}$ a impliqué une hausse d'environ $15 \mathrm{~cm}$ correspondants à une marche qui se conserve encore in situ (Fig. 2, a). On n'a pas détecté des indices d'ouvertures vers l'extérieur de l'espace B, ni dans la paroi nord de l'espace A.

Un autre passage existerait certainement à l'ouest (Fig. 2, ouverture 3), donnant accès à l'espace clos et pavé de mosaïque qui existait où aujourd'hui se trouve le parvis de l'église (Fig. 2, espace C). Si l'on peut confirmer l'existence de ce passage interne, elle gagnerait un écart d'environ $45 \mathrm{~cm}$, l'équivalent a trois marches similaires à ce qui sépare les autres deux compartiments.

Une fois situé sur le parvis de l'ouest de l'église actuelle (Fig. 2, espace C), les inconnues s'aggravent pour plusieurs raisons. C'est, sans aucun doute, un espace qui comprend l'ensemble original et était fermé et couvert. Des fragments de la mosaïque, qui aurait une composition géométrique semblable à l'espace $\mathrm{A}$, le prouvent. 


\section{Analyse descriptif des mosaïques du parois et l'église de Santa Maria do Freixo}

L'élément de patrimoine le plus important de l'ensemble archéologique exhumé au cours de l'intervention archéologique dans le cimetière et l'église de Santa Maria do Freixo est, sans aucun doute, l'ensemble des pavements en mosaïque couvrant, initialement, au moins trois compartiments distincts (Fig. 2, A, B et C).

Pour donner une idée de ce qu'aura été la zone pavée de mosaïque, de ce qui est arrivé à nos jours et ce qui a déjà été exhumé par des fouilles archéologiques (Fig. 3), il suffit d'analyser le tableau suivant (Tableau 1) :

\begin{tabular}{|l|l|l|l|l|}
$\begin{array}{l}\text { Espace } \\
\text { cffr. Fig. 2) }\end{array}$ & $\begin{array}{l}\text { Aire totale } \\
\text { évaluée }\left(\mathbf{m}^{2}\right)\end{array}$ & $\begin{array}{l}\text { Fouilles - } \\
\text { Aire totale }\left(\mathbf{m}^{2}\right)\end{array}$ & $\begin{array}{l}\text { Aire totale de } \\
\text { mosaïque } \\
\text { préservée }\left(\mathbf{m}^{2}\right)\end{array}$ & $\begin{array}{l}\text { Aire totale de } \mathbf{m o -} \\
\text { saïque préservée \% } \\
\text { de l'aire totale }\end{array}$ \\
\hline A & 89,64 & 62,3 & 8,89 & 9,93 \\
\hline B & $>17,5$ & 12,4 & 0,8 & \\
\hline C & 107,45 & 92,2 & 0,12 & 0,11 \\
\hline TOTAL & $>214,3$ & 166,9 & 9,81 & \\
\hline
\end{tabular}

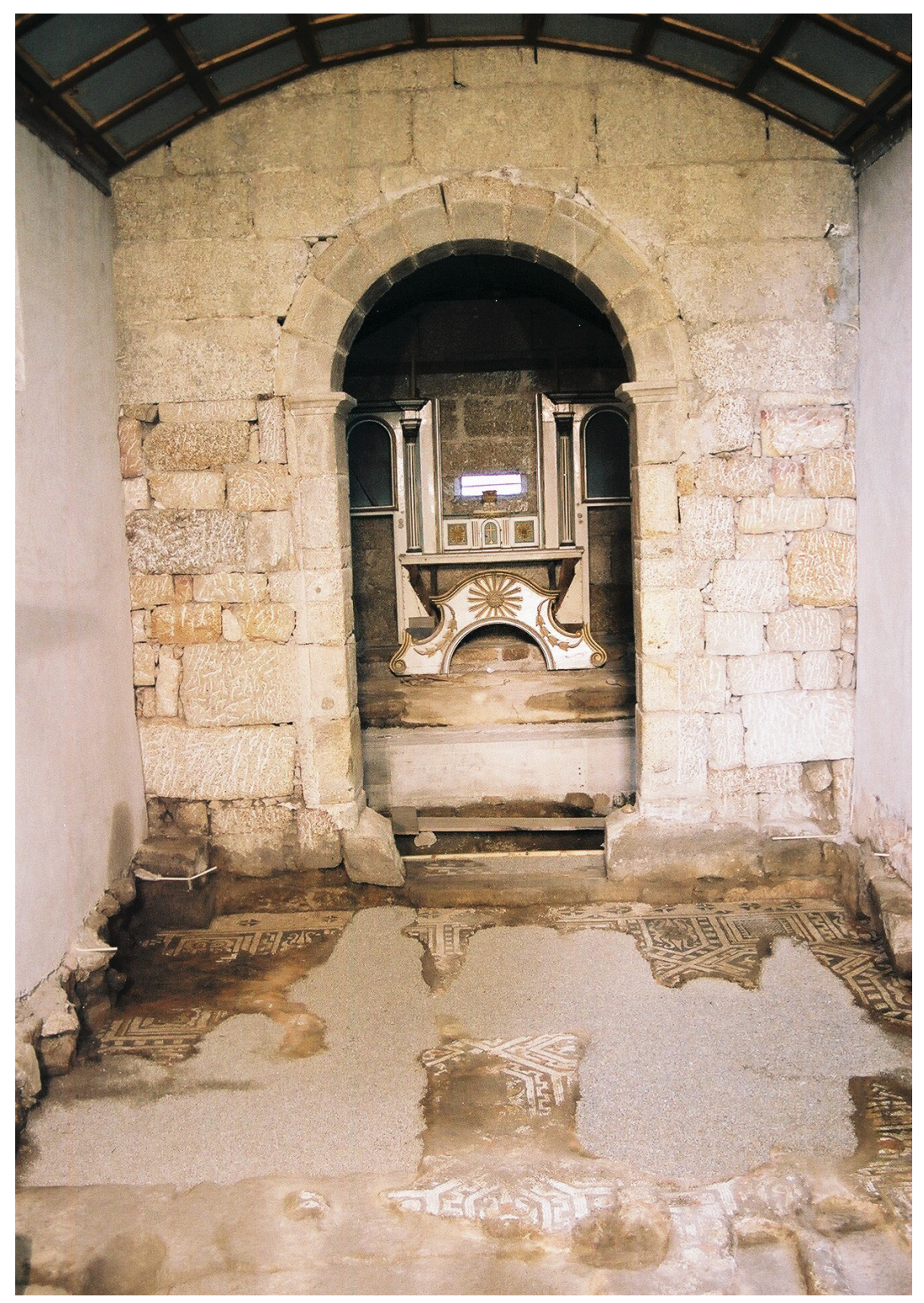

Tableau 1

Bâtiment avec des mosaïques. Aire totale, aire fouillée et aire de mosaïque préservée.
Figure 3

Panoramique de la nef et sanctuaire de l'église de Santa Maria do Freixo au final des fouilles 


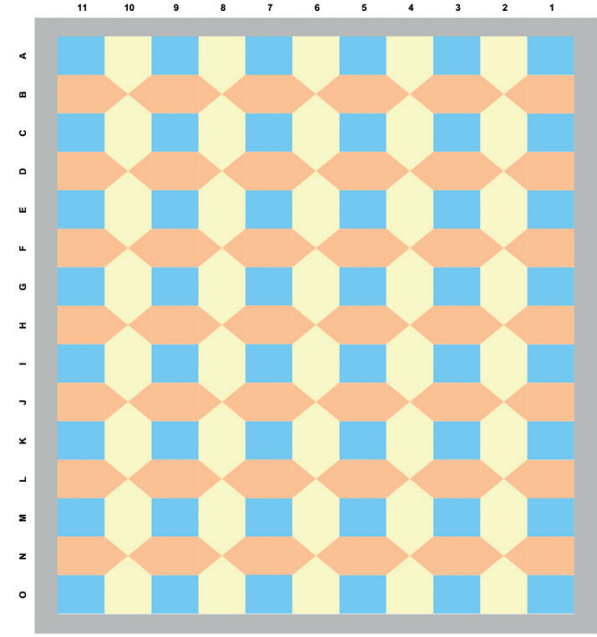

Figure 5

Proposition de reconstitution schématique de la composition orthogonal de la mosaïque de l'espace A

Figure 4

Plan de l'église de Santa Maria do Freixo avec dessin des mosaïques identifiées a la nef, sacristie et sanctuaire (échelle originale du dessin $1: 1$ )
Comme on peut observer, d'une superficie totale estimée supérieure à $214,5 \mathrm{~m}^{2}$, la surface totale de mosaïque dont nous disposons actuellement n'arrive pas aux $10 \mathrm{~m}^{2}$. Et, si dans le cas de l'espace A les vestiges nous permettent de faire quelques considérations sur la composition, l'organisation et patrons thématiques présents dans la mosaïque $\mathrm{B}$ et $\mathrm{C}$ est beaucoup plus complexe.

Pour l'analyse formelle des mosaïques on a utilisé les champs descriptifs de la fiche utilisée par Cristina Fernandes de Oliveira pour la publication des mosaïques de la Villa romaine de Rio Maior (Oliveira 2003: 35-37), qui, à son tour, repose sur la fiche utilisée pour les mosaïques de Torre de Palma (Lancha - André 2000). Cette même analyse est basée sur le dessin de toutes les parcelles de mosaïque conservée, dont l'original a été réalisé par décalque à l'échelle naturelle sur des feuilles de pvc directement appliquées sur la mosaïque.

\section{L'espace A (Fig. 2, A)}

Espace pavé de mosaïques polychromes sur calcaire (Fig. 4). Composition orthogonale avec octogones irréguliers, sécantes et adjacents, qui déterminent carrés et hexagones oblongs (Décor I: pl.169d) (Fig. 5).

Cet espace (Fig. 2, A) correspond à presque la totalité de la nef de l'église actuelle. Dans son axe Nord-Sud il est plus grand que la nef, couvrant une partie de l'allée de l'église (à Nord), le clocher et l'escaliers d'accès respectifs, niveau d'accès à la porte du côté sud de l'église et la sacristie et l'angle NE de la sacristie (à Sud). Ce panneau de mosaïque aurait initialement 50 palmi $(=11,00 \mathrm{~m}) \mathrm{de}$ longueur (Est-Ouest) par 37 palmi (=8,14 m.) de largeur (axe Nord-Sud).

Il était possible de documenter la stratigraphie de la chaussée elle-même dans une place où la même avait été coupée par une tombe creusée dans la roche (Fig. 6). Au moins trois strates distinctes ont été délibérément placés pour constituer le fondement sur lequel s'a installé l'opus tesselatum (Fig. 7). En ce qui concerne la mince couche de terre noire qui s'étend directement sur l'affleurement granitique, cela peut indiquer que la surface qu'elle couvre existait déjà et

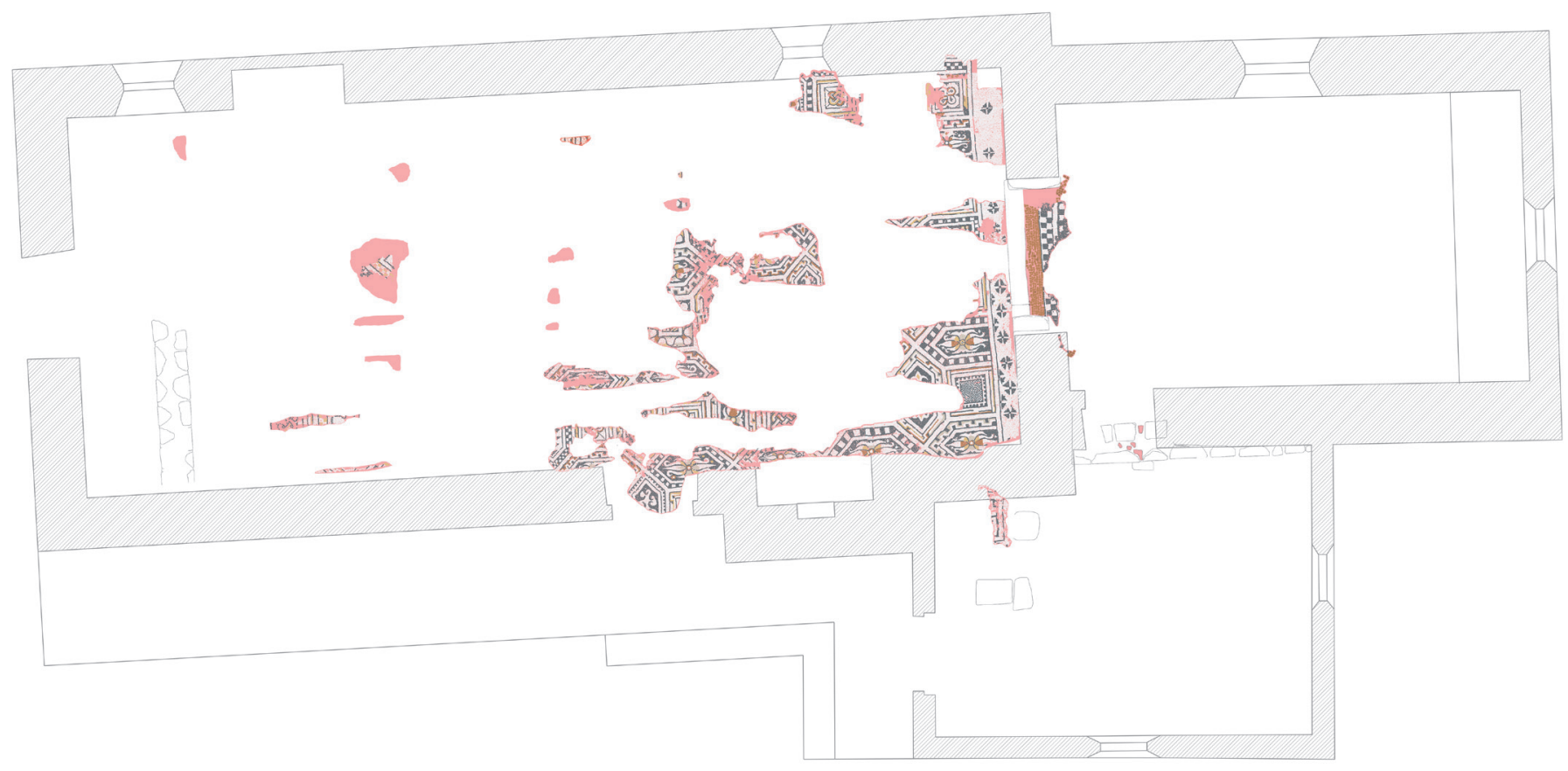




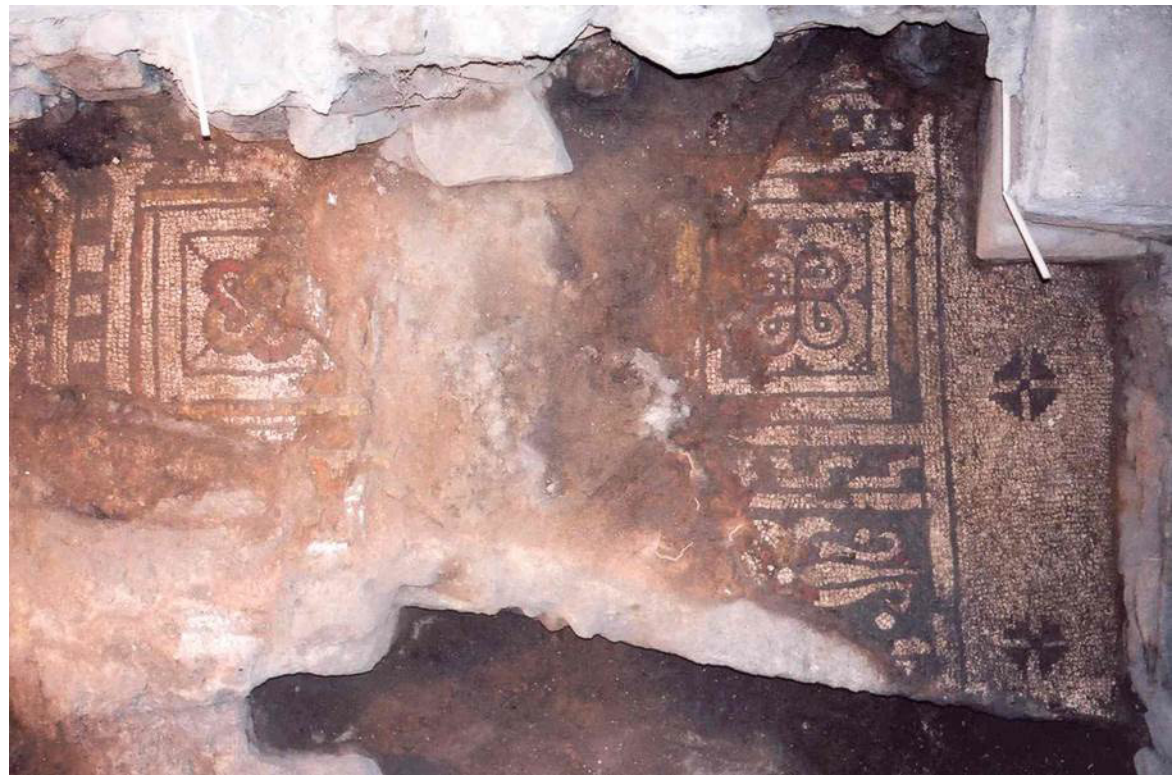

Figure 6

Coin nord-est de la nef de l'église : détaille de la mosaïque coupée par sépulture excavée en la roche

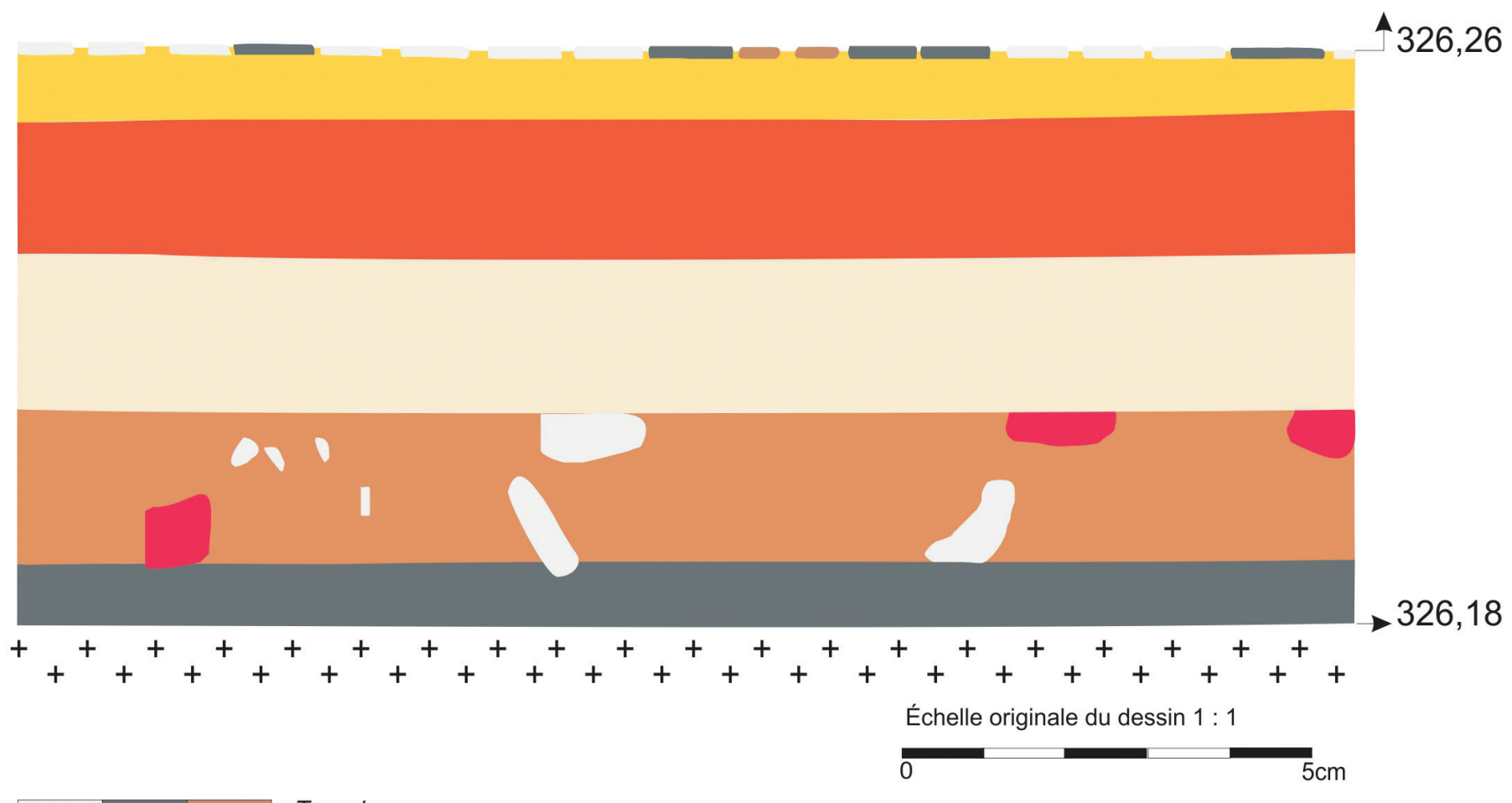

\section{Tesselae}

Couche de pose des tesselae, beaucoup compacté et avec coloration jauni

Couche de preparation, composée par argile desagrégée, et avec coloration châtain-rougeâtre

Couche de preparation, composée par matérial semblable à argile cuite et moulu, desagrégée, et avec coloration blanchâtre

Couche de preparation, composée par terres glaises, très compactes, avec nombreux fragments de ceramique de construction et nodules de mortier

Nodules de mortier

Fragments de ceramique de construction

Couche de terres noires, compactes, avec beaucoup de racines

${ }_{++}^{++}$Affleurement granitique

Figure 7

Coup stratigraphique illustrant la technique de construction de la mosaïque, obtenu à la nef de l'église de Santa Maria do Freixo 
ferait partie d'une construction antérieure déjà abandonnée, dont la couverture de végétation existante était brûlée avant de recevoir la mosaïque.

Nous avons identifié des tesselles en cinq couleurs de base avec quelques variantes, qui, selon le tableau de Munsell (Munsell 1992), ont les dénominations suivantes :

Trois nuances de rouge : rouge jaune (5 YR 5/6), rouge pale (7,5 YR 3/3) et rouge (2,5 YR 4/6); jaune-olive (5 Y 6/7); vert olive (5 Y 5/4); blanc (2,5 Y 8/0); gris foncé (7.5 R 4/0). Contrairement aux deux autres espaces, où les tesselles céramiques ont été utilisées, ici ont utilisé seulement des tesselles de calcaire.

De la périphérie vers le centre, ce tableau est composé des éléments suivants :

Bande blanche avec une ligne de fleurs noires en diagonale croisée (Décor I: 30 pl. $4 \mathrm{j}$ ), pas proches et avec distances irrégulières, majeures dans la moitié nord (44 cm.), plus petits dans la moitié sud (entre 13 et $24 \mathrm{~cm}$.), où la seule ligne de petites fleurs se transforme ponctuellement en double ligne (Fig. 8). De cette bande, nous avons seulement témoignage dans la paroi est et dans le commencement de la paroi sud, en déduisant leur existence aussi au long des parois nord et ouest. Ensuite on trouve une ligne grise foncée simple et une autre blanche et quadruplé.

Au long de la paroi sud il y a une deuxième bande, entourée d'un double filet gris foncé et rempli de blanc dans la réduite superficie visible (Lima 2016a: 112). Dans cette deuxième bande, nous ne savons pas s'il n'y aurait des motifs géométriques ou végétal.

Tout ce cadre fait partie d'une composition orthogonale d'octogones irréguliers, adjacents et sécants, qui déterminent des carrés et des hexagones oblongs. Près des parois, la dernière disposition d'hexagones se transforme en pentagones pour la suppression de l'un des triangles supérieurs, afin d'obtenir une fin linéaire pour l'ensemble, qui se forme par les éléments suivants :

-Est-Ouest, 5 bandes de 1 pentagone +7 hexagones +1 pentagone (Fig. 5 , colonnes 2, 4, 6, 8 et 10), remplis de motifs végétaux. Perpendiculairement à celles-ci, en direction Nord-Sud, 8 bandes de 1 pentagone +4 hexagones +1 pentagone (idem, B, D, F, H, J, L, N, P), remplis de motifs similaires.

Hexagones et pentagones ont un fond gris foncé, entouré de triple filet blanc avec armature qui varie au cas par cas. A l'intérieur ils sont fleuronnés avec une corolle, composites, allongés, avec un bouton central circulaire du quel émergent quatre ensembles d'éléments non contigus : deux éléments, opposés, sont des calices de fleurs de lotus, trifides ; les deux autres, également opposés, pointant vers les sommets de l'hexagone, sont en calice avec des pétales fusiformes et feuilles en crochet (Fig. 9). Apparemment, seulement pour économiser de l'espace, les fleurons qui décorent les hexagones qui forment les bandes N-S sont plus compressés que les perpendiculaires à eux. Cette compression du motif fait que les pétales et les feuilles, dans ces cas (B3, D5, D7 et F3) se fusionnent en un seul élément dès la base au calice, créant deux nouvelles fleurs de lotus.

Les bandes d'hexagones $\mathrm{H}$ et $\mathbf{J}$, c'est-à-dire ceux qui marquent l'axe central de l'entrée dans ce compartiment, utilisent différents motifs dans le remplissage des hexagones : en $\mathrm{H} 3$ on rencontre un motif inconnu avec une quadruple dentelé sur un côté. En J3, c'est apparemment les restes d'une ramure d'où émane un ensemble de feuilles stylisées peltées (Fig. 10).

Dans presque tous les hexagones et pentagones, il y a toujours de petits éléments détachés inscrits sur le fond noir, en contact ou non avec les extrémités des 


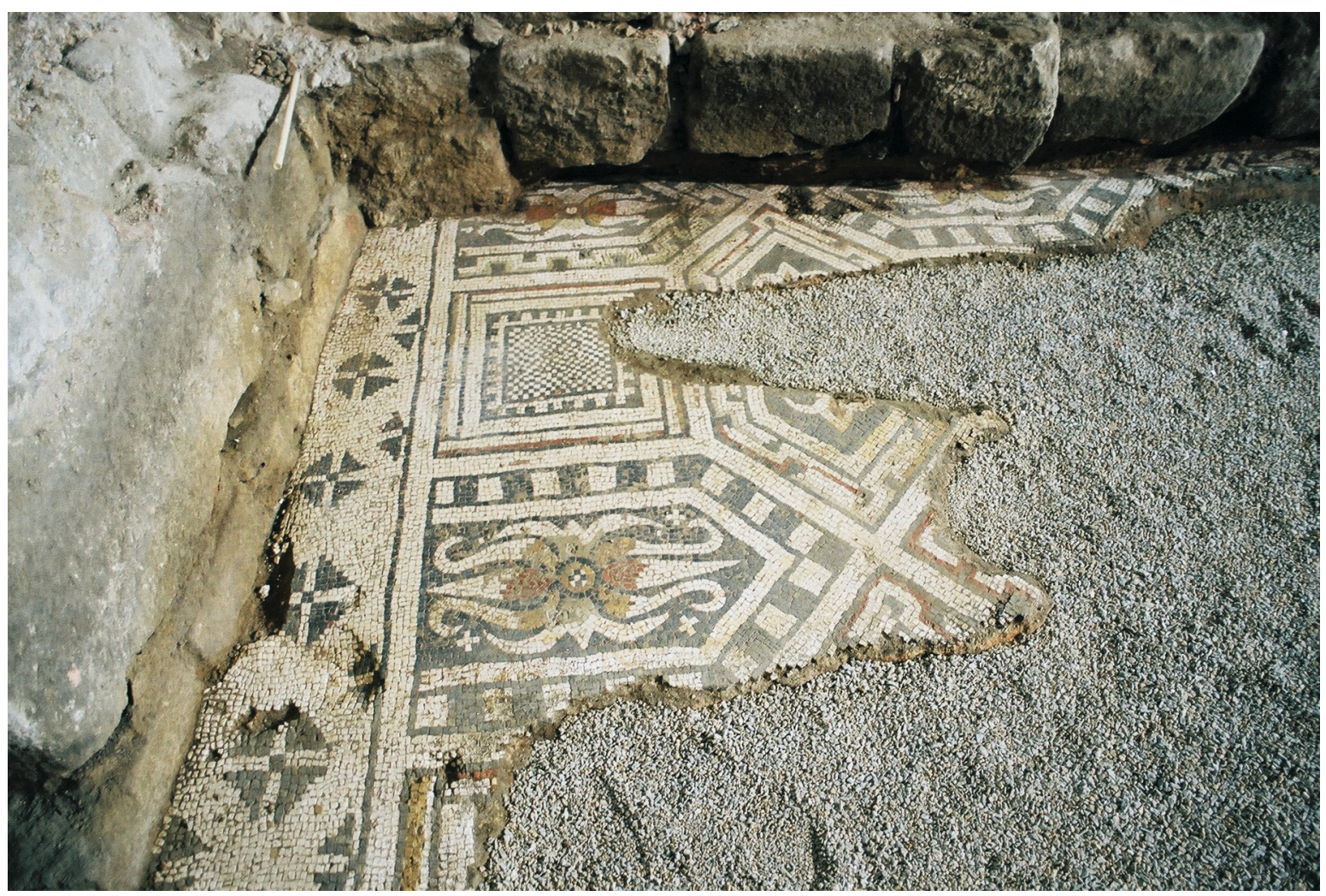

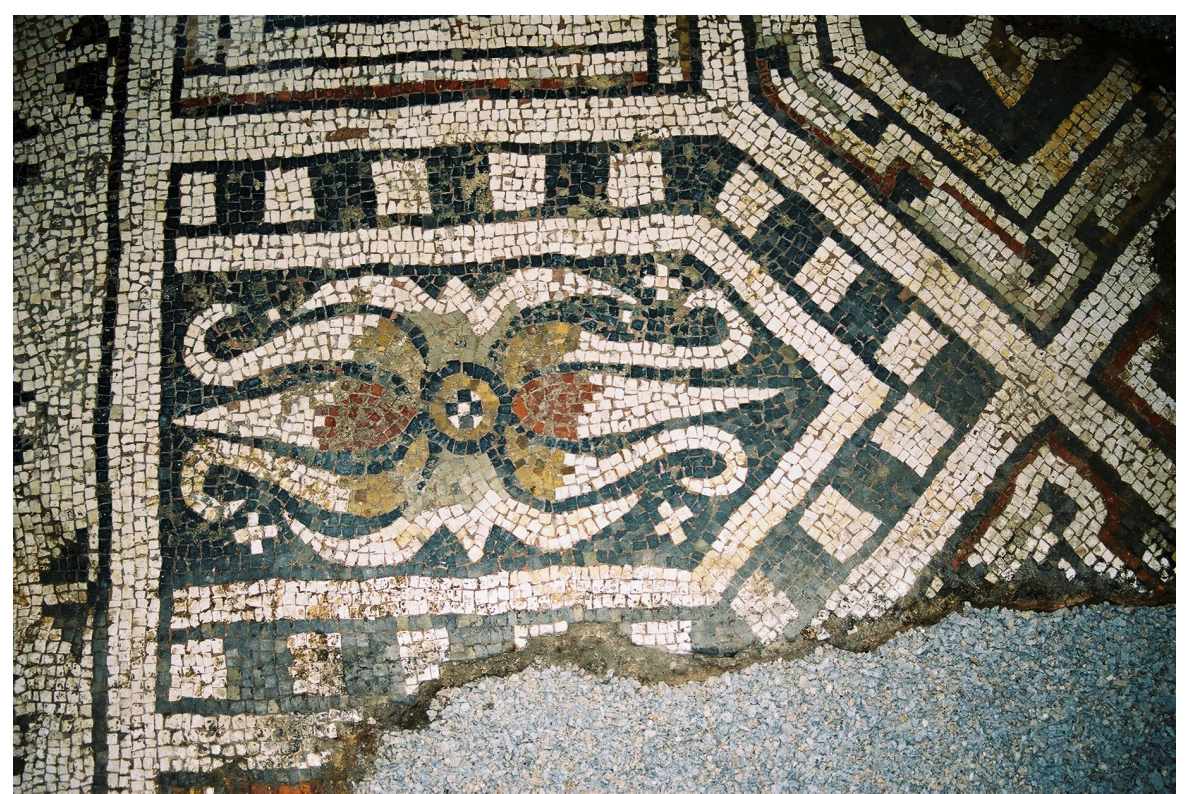

feuilles. Ainsi, A2 révèle une simple croix blanche, bien que l'objectif pourrait être la création de trois croix, dont deux ont abouti seulement à petites figures indéfinis. L'A4 le suggère en comportent trois croix identiques avec une disposition similaire. A6 conserve seulement deux croix identiques mais inscrites sur fond coloré, un jaune et l'autre blanc. A8 ne révèle que deux cercles simples remplis de blanc. En $\mathrm{C} 2$ on retrouve encore deux croix simples, blanches, et des traces d'une troisième croix. En G2, trois cercles, deux remplis en jaune et un troisième semblable au bouton central du fleuron, blanc avec érable rouge. G4 reprend les trois croix simples de tesselles blanches.
Figure 8

Coin sud-est de la nef de l'église bande double de petites fleurs en croix et le principe de la composition orthogonale

Figure 9

Bout de la composition qui rempliait

l'espace B, proche de la marche de passage pour l'espace A. Sanctuaire et nef de l'église de Santa Maria do Freixo 
Figure 10

Détaille de l'axe central du panneau de mosaïque qui couvrait l'espace A. Cet axe correspond à l'actuel accès sud à la nef de l'église de Santa Maria do Freixo

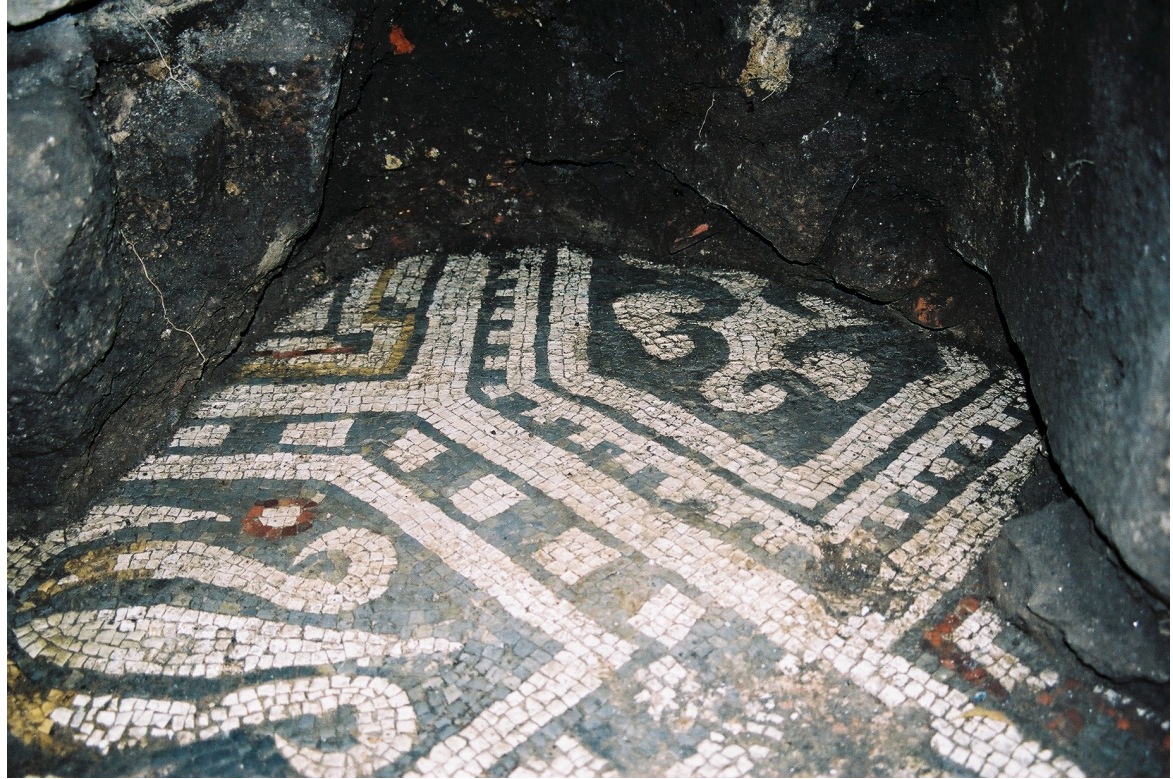

En ce qui concerne les cadres de ces figures géométriques, nous avons identifié cinq types différents, avec des variations au niveau du traitement chromatique sur deux d'entre eux: 1) la bande de carrés adjacents avec tesselles 4 x 4, alternés gris foncé et blanche; 2) la double bande détournée de carrés adjacents avec tesselles 4x4, alternés gris foncé et blanc ou jaune et blanc; 3) la bande de double dentelé bordé par filet blanc dans le dentelé à l'intérieur et du fil coloré dans le dentelé extérieur; 4) la bande composée de segments allongés de méandre coloré, alternativement jaune/rouge/vert sur fond blanc, qui se croisent formant sommets internement occupés par des triangles gris foncé; 5) le filet dentelé simple en groupes de quatre tesselles.

Hexagones et pentagones, ensemble, définissent un total de 54 (6 x 9) champs rectangulaires dont nous ne connaissons, total ou partiellement, que 14.

De ces champs, seulement en 9 il est perceptible le motif représenté (A3, A9, C9, E3, G3, G5, G7, I3 et O3). Dans ces 9, nous avons au moins 7 motifs différents. $\mathrm{Il}$ y a un motif qui se répète avec certitude $(\mathrm{C} 9 / \mathrm{O} 3)$ et un autre dont la répétition, bien que possible, n'est pas entièrement sûre, compte tenu de l'état de destruction des deux (G5/G7). Ces sept motifs sont :

En A3: échiquier (Fig. 11), composé de carrés adjacents, tour à tour gris foncé et blanc, avec remplissage très irrégulière et des lacunes. L'échiquier est composé d'un carré avec 20 tesselles par côté, bordé, successivement, par un fil gris foncé simple, un fil blanc simple et un filet dentelé d'éléments irrégulièrement carrés. Le cadre extérieur de cet échiquier est similaire à celle de presque tous les autres champs quadrangulaires.

En A9 : Quadrilobe entrelacée (Blanchard et al. 1973: 25 n. ${ }^{\circ}$ 61) (Fig. 12). Sur un fond blanc, figure quadrilobée, entrelacée, avec tous les lobes avec remplissage blanc et délimitée par un il gris foncé. Deux des lobes, opposés, ont aussi un fil rouge - simple sur un côté, double de l'autre. Le quadrilobe est au centre d'un quadrilatère gris foncé, dont les sommets et le centre de chacun de ses côtés ont dessinés des triangles avec trois tesselles par côté, respectivement dans les échecs et en pleine, créant une projection de la figure trilobée pour l'extérieur (Fig. 13). Le cadre de ce champ est identique à celui de I3. 


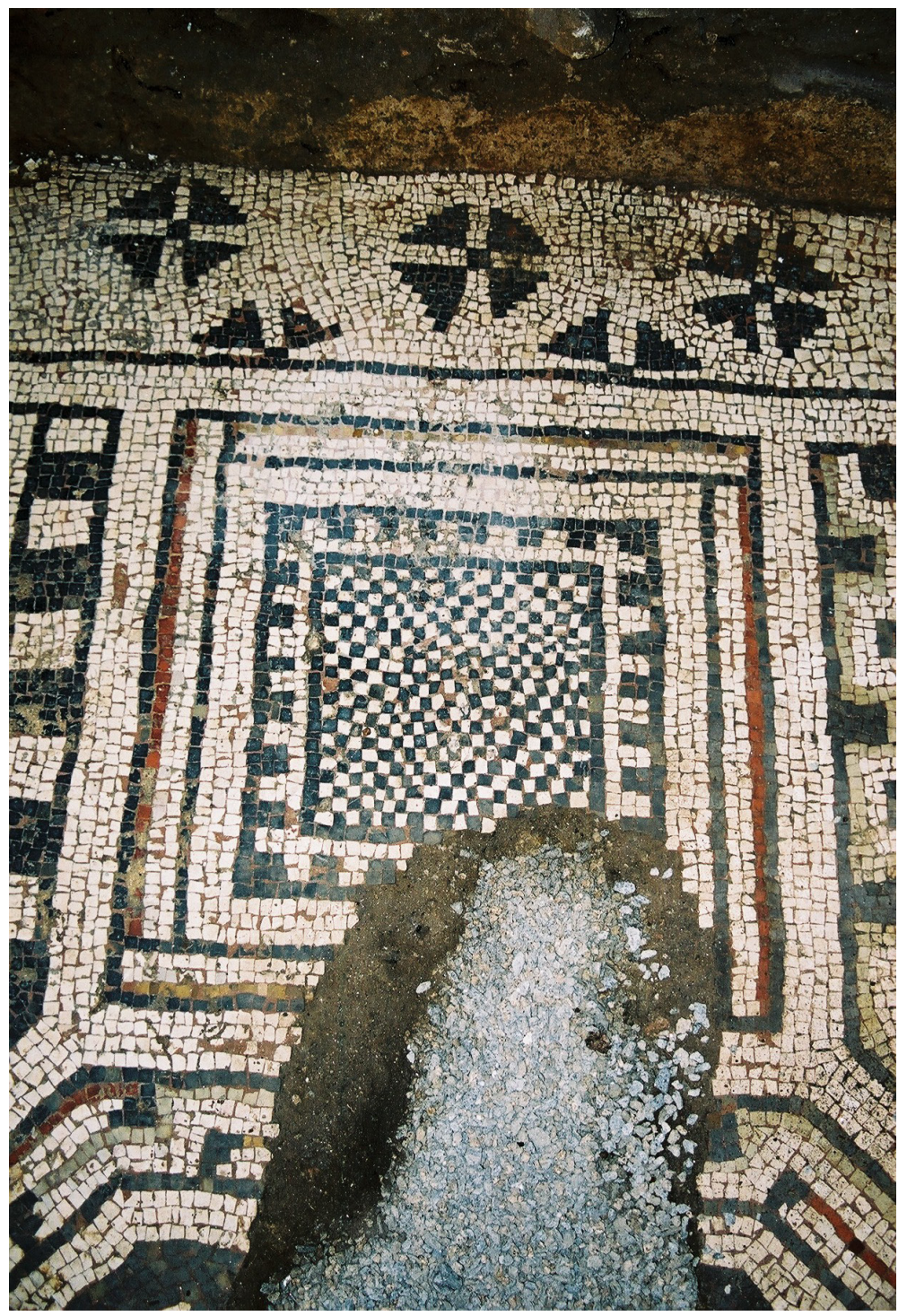

Figure 11

Échiquier (champ quadrangulaire A3). Nef de l'église de Santa Maria do Freixo

Figure 12

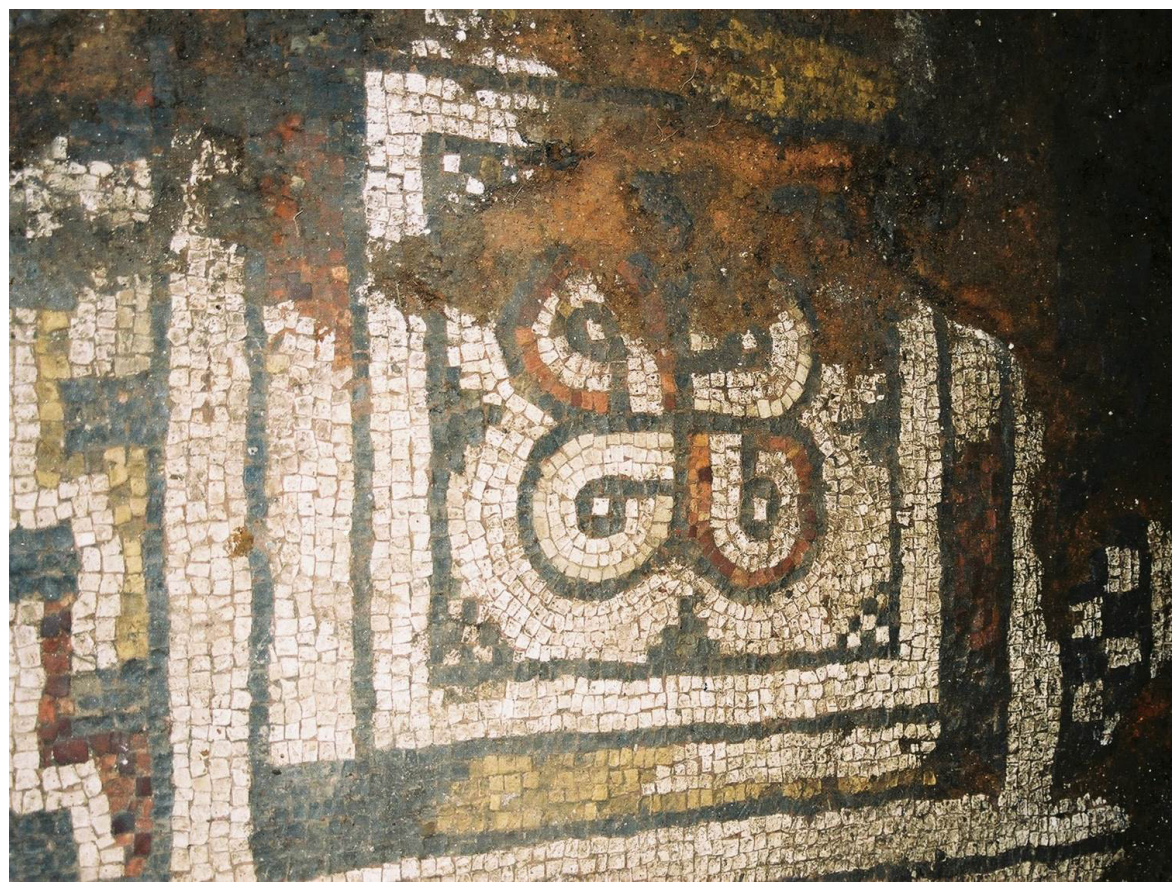

Quadrilobe entrelacé (champ quadrangulaire A9). Nef de l'église de Santa Maria do Freixo 
Figure 13

Détaille du coin nord-est de la nef de l'église où s'observe le pavé en mosaïque : gros plan d'une figure quadrilobée, fleuron et bande de petites fleurs en croix, adossant à un socle granitique contemporain de la mosaïque

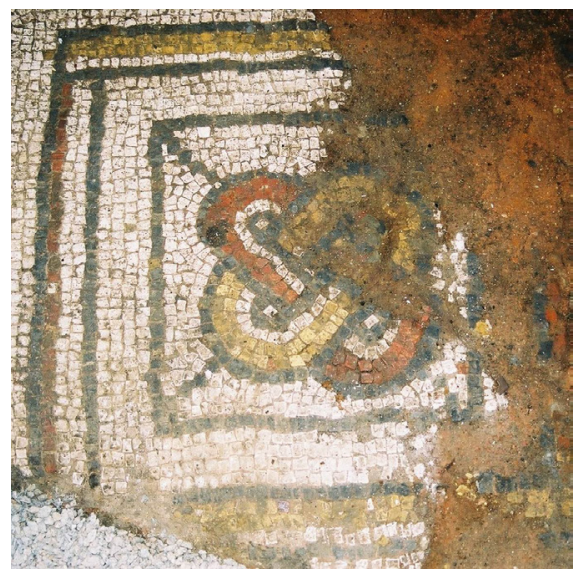

Figure 14

Nœud de Salomon (champ quadrangulaire C9). Nef de l'église de Santa Maria do Freixo

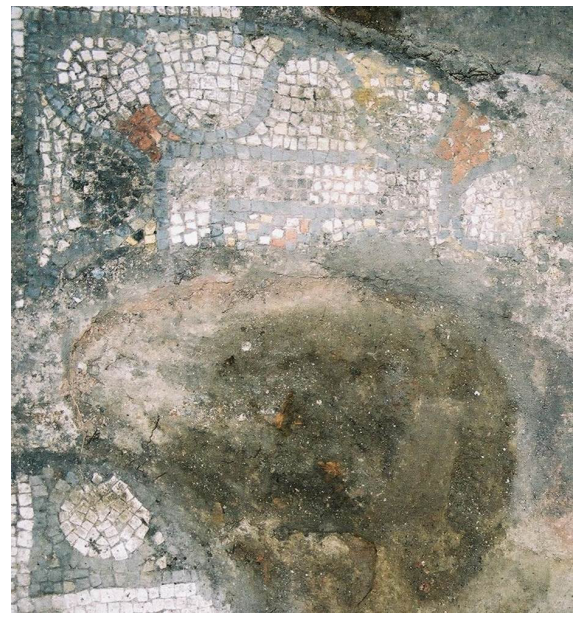

Figure 15

champ quadrangulaire G5 avec cadre aux ondes. Nef de l'église de Santa Maria do Freixo

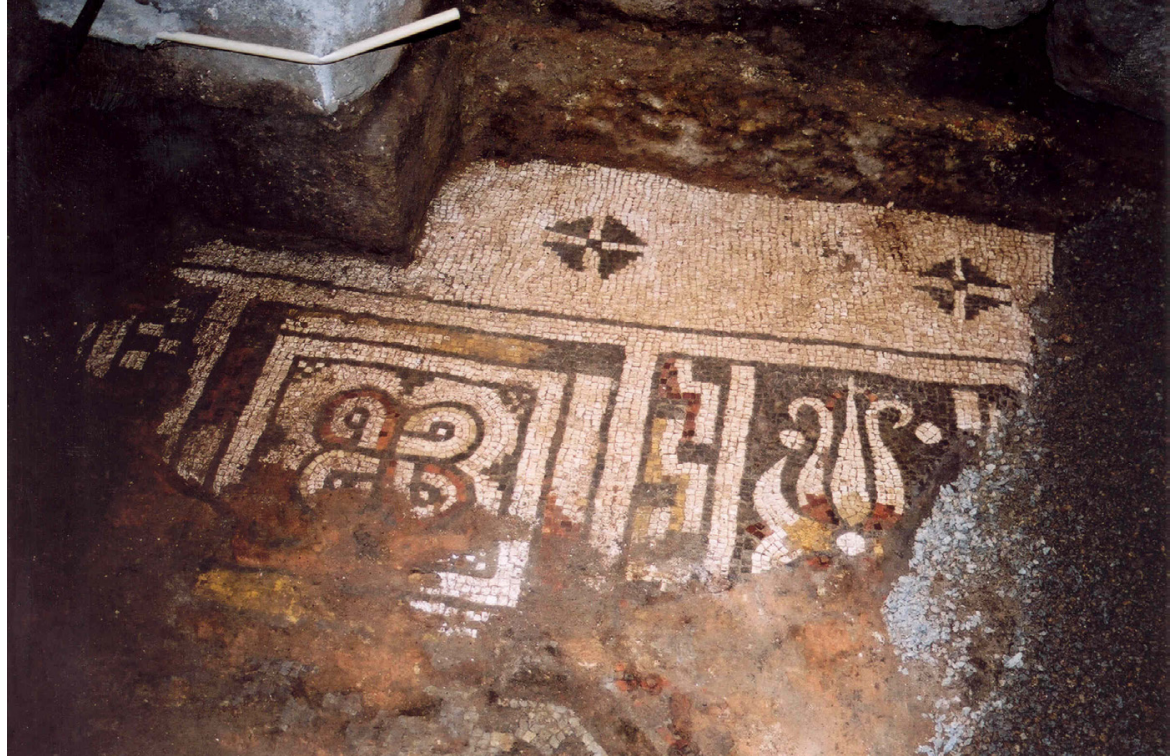

coloré : dans le cas de crochets allongés, rouges dans un cas, vert dans l'autre; dans le cas du crochet rectangulaire, le fil est jaune. Le cadre extérieur de ce champ est similaire à celui de la plupart des champs rectangulaires.

En G3: Champ qui contient une figure géométrique dont il y en a qu'un lobe ou un crochet, démarqué par une succession de quatre lignes, successivement gris foncé, rouge, blanc et gris foncé encore une fois, tous simples à l'exception du blanc qui est double, autour d'un centre remplis de pièces blanches. Ce figure avance sur le quadrilatère qui l'encadre, et ceci est délimité par un filet simple gris foncé et remplis par deux autres, un jaune, simple, et l'autre blanc, double.

En G5/G7: il est commun à ces deux champs la présence d'une bande de vagues polychromes délinées gris foncé (Fig. 15), qui bordent un carré central. En G5, les vagues des sommets ont un remplissage blanc/rouge tandis que les côtés ont une alternance blanc/jaune et blanc/vert. G7, on a seulement deux vagues à deux sommets, le jeu des couleurs pourrait être le même, mais on peut seulement attester la présence du jaune au début de l'un des ondes latérales. Du champ central carré, rien est observé dans le G7. Mais en G5 s'est conservée une petite partie de celui-ci, délimité du gris foncé et remplis par des lignes diagonales formant les séquences gris/blanc/jaune et gris/blanc/rouge (et encore une fois gris/blanc/jaune ?)

En I3: Dans ce champ, duquel seulement la moitié est conservée, est représentée une figure apparemment quadrilobée dont s'observent trois lobes. Si elle représente la même figure qui apparaît représenté sur l'A9, son orientation est différente et, comme le nœud de Salomon présente en C9, elle a les mêmes lignes simples en diagonale gris foncé sur fond blanc, unissant la figure aux sommets du carré encadrent. Le cadre extérieur de ce champ est identique à ce qui est présent à $\mathrm{A} 9$.

En ce qui concerne les cadres des champs carrés, nous avons identifié quatre types différents : 1) est le plus commun, présent dans 8 des 13 champs dans lesquels il était possible de l'identifier (A3, A5, A7, C9, E3, E5, G3 et O3). Dans un carré délimité par un filet gris foncé, deux côtés opposés reçoivent un filet simple jaune ou double et un autre blanc, toujours double. Les deux autres côtés ont la même séquence, remplaçant le jaune par le rouge. Fermant intérieurement 
le cadre deux autres filets, un blanc, triple, et un autre gris foncé, simple; 2) est présent dans deux autres domaines (A9 et I3) et se distingue de la précédente en raison du filet blanc, du jaune et du rouge, à triple filet, occupent tour à tour la moitié de chaque côté du carré qui délimite la champ central et le carré a toujours les sommets remplis de gris foncé; 3 ) est présente dans deux autres champs (G5 et G7): bande de vagues polychromes délinéés de gris foncé, déjà décrites; 4) dans le cadre du champ G1 nous avons identifié un type non présent dans les autres champs carrés mais très commun dans les hexagones : la bande composé par des segments allongés de méandre coloré, alternativement jaune/rouge (et probablement aussi vert), sur un fond blanc, qui croisent formant des sommets remplis par des triangles gris foncés.

\section{Espace B (Fig. 2, B)}

La très petite zone conservée ne permet pas de reconstruire le thème présent dans ce compartiment. Et étant donné que cet espace n'a pas été entièrement fouillé ni défini, nous pouvons estimer seulement ses dimensions. Leur profondeur ne serait pas moins de 3,9 m. Sa largeur aurait au moins 4,5 m.

Dans la région fouillée, seule une petite partie de la chaussée contre le seuil de passage entre la nef et le sanctuaire, sous l'arc triomphal de l'actuel église, est conservée. Au total, sont conservés seulement environ $8 \mathrm{dm}^{2}$ de pavement en mosaïque.

Au-delàs des tesselles blanches et gris foncée, identiques à celles utilisées dans l'espace A, ont été utilisées tesselles d'argile, comme dans le compartiment C.

En s'appuyant au seuil qui a servi de marche pour le passage entre les espaces A et $B$, il est possible d'observer qu'une bande de tesselles céramiques suivie d'une double bande mixte de carrés adjacents, alternant le fris foncé et le blanc (Fig. 16).

Ce qui s'observe en suite sera déjà le début d'une figure géométrique dont nous ne connaissons pas son remplissage. Aurait un cadre similaire à celle de certains hexagones et pentagones de l'espace A, avec une double bande de carrés adjacents, alternant la gris foncé et le blanc.

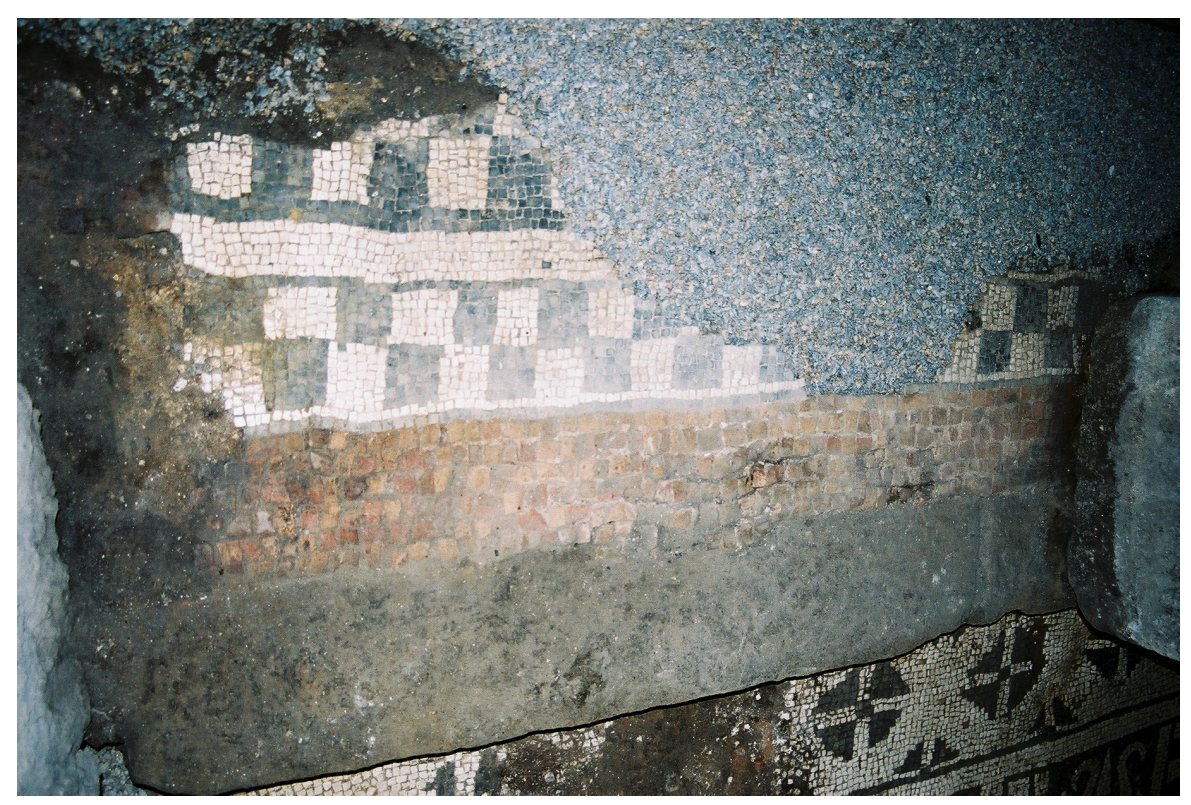




\section{Espace C (Fig. 2, C)}

Un petit fragment semble indiquer un motif semblable à ce qui remplit les hexagones/pentagones de l'espace A, et nous avons mis la possibilité d'être une composition similaire. Aurait la même largeur que l'espace A (37 palmi = 8,14 mètres) pour environ 60 palmi (13,2 mètres) d'axe E-W.

En plus des tesselles blanches et gris foncées, identiques à celles utilisées dans le compartiment $\mathrm{A}$, ont utilisé des tesselles d'argile, comme dans le compartiment B.

Dans un fragment, s'observe la bande de tesselles en céramique qui, comme l'espace B, marquait la fin de l'extérieur de la composition. Dans un autre fragment, sont seulement visibles 20 tesselles blanches, qui, par sa position relative, pourraient appartenir à une des bandes qui sont observées également en l'espace $\mathrm{B}$. Le fragment identifié au milieu du parvis de l'église, bien que très petit, nous montre clairement ce qui reste d'un fleuron semblable à ces qui remplit les pentagones et hexagones, qui font partie de la composition présente dans l'espace A.

Si cette hypothèse se confirme, cet espace pouvait étaler une composition orthogonale très similaire à celle observée dans la nef de l'église, en s'adaptant à la plus grande longueur de l'espace disponible. Au total, le compartiment aurait alors, en direction Est-Ouest, 5 bandes avec 1 pentagone +9 hexagones +1 pentagone ; perpendiculairement à ceux-ci, en direction nord-sud, 8 bandes de 1 pentagone +4 hexagones +1 pentagone.

\section{Les mosaïques et son contexte historique et géographique : quelques réflexions}

La découverte de mosaïques romains dans le sous-sol des églises du Nord de Portugal et Galice n'est pas une nouveauté absolue, bien que les exemples connus puissent correspondre à des bâtiments et pavements avec une typologie et chronologie très diverse, soit distinct de ce à Tongobriga (Regueras Grande 2013), soit, probablement, très semblables (Fontes 2009).

Les mosaïques découvertes sous l'église de Santa Maria do Freixo ont été l'objet d'une première étude, datée de 2010, publiée en 2012 (Lima 2012) et très récemment publiée à nouveau (Lima 2016b).

Dans cet étude, nous signalons la possibilité d'être en face d'une maison, c'està-dire, une domus romaine, tardive, mais nous n'oublions pas de signaler les questions que cette interprétation soulève et de faire des suggestions alternatives émergeant de ce qu'on connaissait sur le contexte historique et archéologique du noyau urbain de Tongobriga à l'époque de sa construction, qui remonte au Vème siècle.

Ce contexte pointe vers l'existence, dans l'antiquité tardive, au local ou dans les environs, d'un premier temple chrétien, condition sine qua non pour Tongobriga comme primitive paroisse de la diocèse portucalensis dans le siècle suivant (David 1947: 30-40). Cela nous a fait penser sur la possibilité du bâtiment découvert sous l'actuel église avoir été adapté a telles fonctions, même quand nous reconnaissons que son plan - non totalement connu - n'avait pas correspondance avec les modèles connus des édifices destinés à un temple (Lima 2012: 44-46).

Il n'est possible, face aux données actuellement disponibles, démontrer de façon conclusive cette hypothèse, en particulier à cause de l'absence de preuves 
irréfutables de la célébration d'actes liturgiques dans l'édifice pavée à mosaïque, soit au niveau du programme architectonique, soit au niveau des matériaux archéologiques. Ce qui nous oblige à pondérer tous les facteurs que on doit tenir en compte pour interpréter les mosaïques et le bâtiment pour lequel elles ont été faites.

Ses facteurs peuvent être groupés en quatre types : l'analyse formelle du plancher; le plan du bâtiment; le contexte local et le contexte régional.

\section{a) Analyse formelle des mosaïques}

La composition orthogonale qui organise la mosaïque de Tongobriga surgit en des horizons géographiques et chronologiques très différents: dans sa version plus simple et dichromatique, apparait déjà à Pompéi et remonte aux origines de l'opus tesselatum (Oliveira 2010: 399) apparait en versions plus élaborées, au moins depuis le IIème siècle en mosaïques de contextes d'habitation romaines d'Hispania (idem, ibidem; Lopez Monteagudo 2002: note 43 et lame 5) et apparait encore dans les panneaux de Ravenne, datés du milieu du VIème siècle (R. Fariolli Campanati, apud Oliveira 2010: I, 401).

Mais les mosaïques de Tongobriga ont clairement des affinités avec le groupe plus tardif de cet ensemble de parallèles, car s'intègrent pleinement dans une, déjà très évoluée, étape d'un processus qui se développe à partir du IIIème siècle, au cours duquel les figures géométriques qui remplissent les carrés se diversifie plus et les hexagones ont tendance à augmenter en taille jusqu'à qu'elle soit égale au côté carré, ce qui leur permet de recevoir la décoration (Oliveira 2010: I, 399).

Parmi les éléments décoratifs que les hexagones obtiennent, et qui se considèrent déjà caractéristiques des mosaïques du IVème siècle et suivants, on compte les fleurons longilignes qui apparaissent a Tongobriga déjà très élaborés et avec un traitement chromatique complexe, ce qui suggère une stylisation résultante de la maturation d'un long processus d'évolution.

En ce qui concerne les champs rectangulaires, brandissent des motifs décoratifs aussi très fréquents dans la mosaïque romaine et romaine tardive. S'il y a une caractéristique frappante dans la mosaïque de Tongobriga, c'est la diversité des motifs utilisés. On ne peut pas autrement que signaler l'utilisation de la paire quadrilobe/nœud de Salomon, dans des modèles très similaires et inséré dans une composition identique à Milreu (Oliveira 2010: II, estampe III); et encore à São Miguel de Odrinhas (Caetano 2008) un contexte généralement interprété comme tardif.

Peu significative pour nous clarifier le contexte historique et chronologie des mosaïques de Tongobriga est aussi la frise de «fleurs en croix», dont l'usage, très rebattue dans la mosaïque romaine pour remplir les bandes de rattachement au mur, ne sert d'indicateur chronologique. Il survient aussi fréquemment à Milreu dans des compositions du IVème siècle (Oliveira 2010: I, 363) et, bien que dans le cadre de la réutilisation, à la reconstruction de la Basilique de São Martinho de Dume et pourrait avoir appartenu à la période du primitif temple de l'époque suève (Fontes 2009: 187 fig. 5).

\section{b) Plan du bâtiment}

Dans l'étude que nous avons publié en 2012, en supposant le caractère tardif des mosaïques de Tongobriga et, par ça, les intégrant chronologiquement dans le 
contexte des vici qui exerçaient le rôle de «paroisses » dans le cadre de l'organisation ecclésiastique du diocèse portucalensis dans la période correspondant au royaume suève, nous cherchons des parallèles avec le bâtiment identifié sous l'église de Santa Maria do Freixo entre ceux qui, pour la même époque, étaient déjà connues ailleurs dans le Nord-Ouest. Nous avons constaté que le bâtiment de Tongobriga n'était pas semblable à aucun d'eux ni correspondait à aucun des modèles connus de l'architecture religieuse paléochrétienne.

En revanche, la possibilité d'être une domus est toujours attractive. Mais la comparaison de ce bâtiment avec les autres exemples locaux de l'architecture domestique trouve un obstacle dans les dimensions de ses compartiments. En effet, sont déjà connus à Tongobriga plusieurs exemples de domus, dont l'architecture est basée sur des modèles importés qui pourraient servir de référence.

Si l'on tient en compte uniquement les espaces couverts des domus, ses cubicula ne dépassent jamais, à Tongobriga, les $12-13 \mathrm{~m}^{2}$ d'aire. Même les plus grands compartiments, comme le possible triclinium de la « Maison de l'Impluvium», arrivent seulement à $19 \mathrm{~m}^{2}$.

Admettant la possibilité des espaces découverts, comme l'atrium des maisons avec patio central, peuvent aussi recevoir pavement de mosaïque et aussi servir comme référence, les grandes zones des domus augmentent considérablement, arrivent aux $45 \mathrm{~m}^{2}$ dans ce qu'on appelle la « Maison du Puits » ou $32 \mathrm{~m}^{2}$ dans la «Maison de l'Impluvium». En tout cas, nous sommes loin des compartiments avec plancher en mosaïque découvertes dans le sous-sol de l'église de Santa Maria, lesquels atteignent des valeurs prochaines ou même le double de quelque autre espace domestique de la Tongobriga romaine - respectivement, $89,54 \mathrm{~m}^{2}$ et $107,45 \mathrm{~m}^{2}$.

Ayant ce facteur en compte, lequel suggère se traiter d'un grand bâtiment placé au centre géographique et topographique du noyau urbain muraillé, pas comparable, en dimensions, aux restants domus à Tongobriga, la possibilité d'être un bâtiment érigé par l'élite local avec d'autres fonctions, pas les fonctions d'une simple habitation, gagne force.

\section{c) Contexte local}

Plus par le contexte archéologique local - en particulier par les espaces qu'il occupe et les transformations qui a impliqué dans le tissu urbain et sur les bâtiments préexistants - que par parallèles artistiques et architecturales, nous ne pouvons pas dater les mosaïques et l'immeuble auquel elles appartiennent d'une époque antérieure au Vème siècle.

Il a été possible de constater que les matériaux qui se produisent dans le fossé de fondation de la paroi Sud de l'espace central (espace A) de l'édifice pavée de mosaïque pointent un terminus post quem pour la construction au début du Vème siècle; et un petit mais significatif verre, recueilli dans une des couches de préparation pour la fixation du même panneau de mosaïque, a une chronologie qui pointe vers le «Bas-Empire ou Haut Moyen Age» ${ }^{1}$.

Ce bâtiment est dans le centre du périmètre fortifié qui a toujours constitué l'espace habité de Tongobriga (Fig. 17); c'est aussi un lieu topographiquement détaché, avec un grand espace utilitaire qui a fait du « rossio » (parvis, place)

1 L'analyse et la classification chronologique du verre en question se doivent à la Dr. Jeannette U. Smith Nolen. 


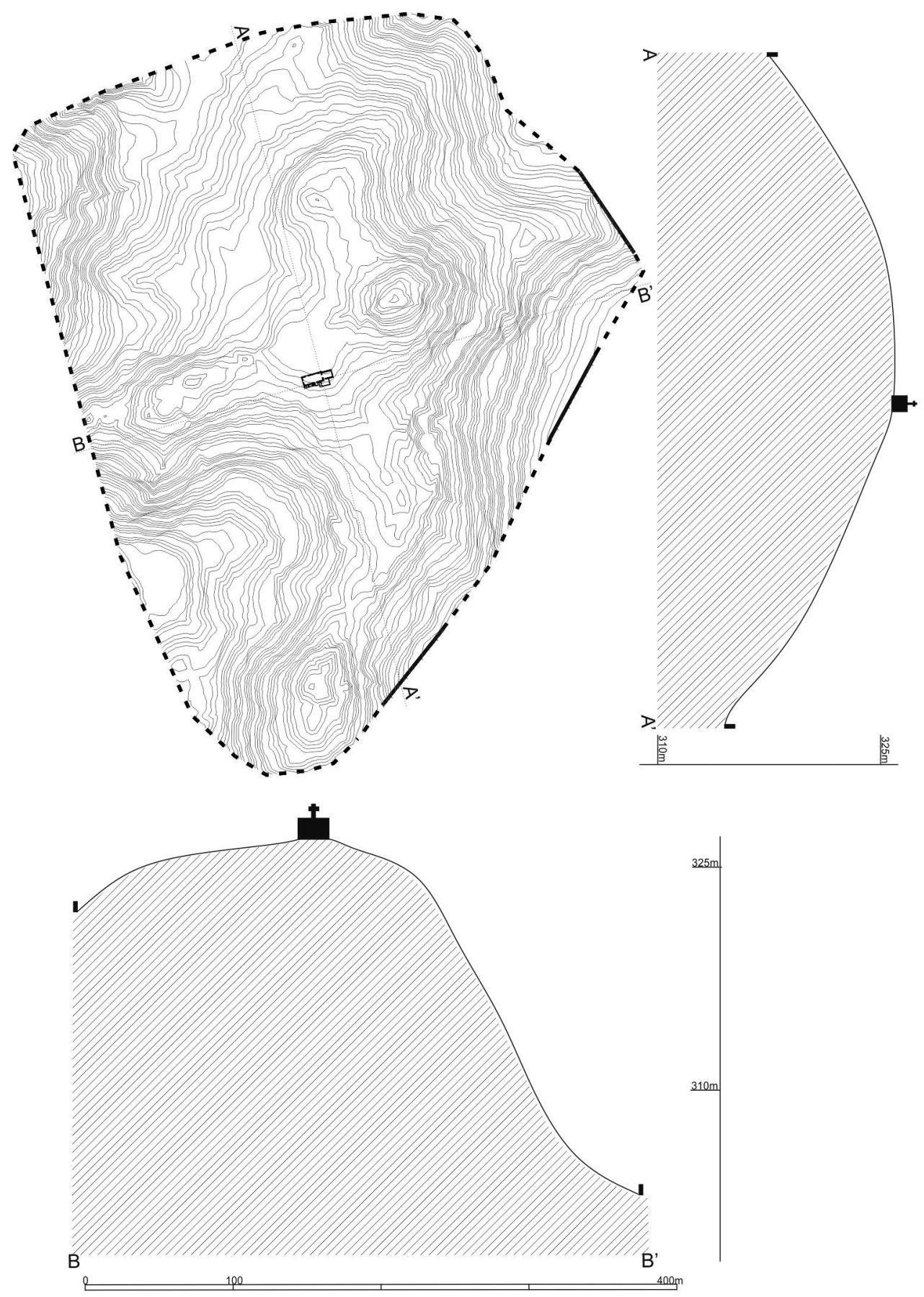

Figure 17

Topographie de l'espace muraillé de Tongobriga avec implantation de l'église de Santa Maria do Freixo (plan et profile)

de l'église une zone de choix: voici comment nous pouvons définir en quelques mots, l'environnement de l'église de Santa Maria (Fig. 18).

Les matériaux provenant de contexte stratigraphique fermé, relatives à la dernière fois d'utilisation d'une domus (appelée «Domus au Sud de l'Église ») partiellement démantelée pour la construction du bâtiment pavé de mosaïque, ne dépassent pas la seconde moitié du IVème siècle ou la première moitié du Vème siècle et, par ça, ce dernier lui doit être postérieur.

La même chronologie (seconde moitié du IVème / Vème siècle) pour les tombes plus tardives qui composent la vaste nécropole « dehors les portes» (Dias 1997: 119-126), qui nous indiquent également un terminus post quem pour les tombes les plus anciennes identifiées dans l'aire de l'église (Lima 2003). Du point de 


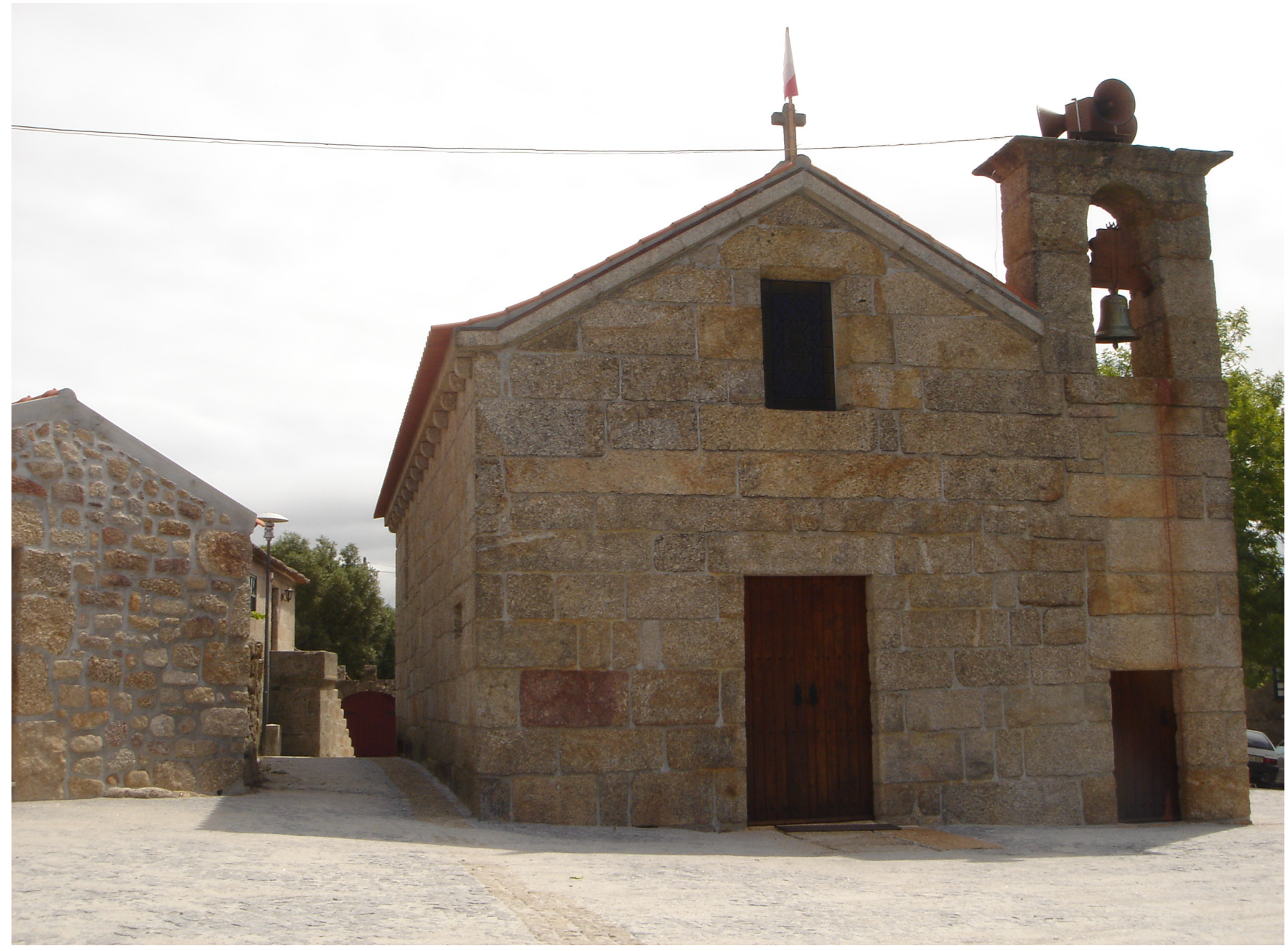

Figure 18

Église de Santa Maria do Freixo (Marco de Canaveses) vu chronologique, cette question est déterminant, sauf si on pose l'hypothèse de, pendant un période indéterminé de temps, avoir eu une utilisation simultanée d'une nécropole «dehors les portes» et un cimetière à l'intérieur de l'espace habité.

La mise en œuvre de l'immeuble qui se trouve sous l'église de la paroisse de Freixo soulève encore un autre problème : celui de la continuité du tissu urbain qui aura orienté le développement des nouveaux bâtiments à Tongobriga au moins depuis le IIème siècle. Ce réseau, dont la mesure standard serait l'actus quadratus, fait de l'exact endroit où ce bâtiment se trouve l'intersection des deux axes (cardus et decumanus maximus ?) (Rocha et al. 2015: 97). Ce qui signifie que l'édification de cet espace comportait un de deux changements structurels : ou une roture avec la stratégie précédente de construction urbaine ou, au moins, la modification de la physionomie dans la zone du noyau central de l'espace habité, ce qui serait conforme à la promotion comme le centre civique du village, au détriment de la zone du forum.

Ce phénomène de la cessation de la capacité (et/ou la volonté) d'investissement dans le forum est guidé vers la seconde moitié du Vème siècle, ce qui est d'accord avec les autres indicateurs chronologiques que nous avons recueillis.

En bref, la simple analyse formelle de la mise en œuvre du bâtiment identifié sous l'église de Santa Maria do Freixo et aussi l'analyse de sa relation avec les bâtiments environnants, et aussi le changement des stratégies de puissance 
qui représente la construction, à cet endroit, d'un bâtiment prestigieux, ouvrage d'une élite locale, remarquent que la mosaïque faire partie d'une intervention urbaine du Vème siècle.

\section{d) Contexte régional}

Vème siècle après J.-C. Conventus bracaraugustanus, tout près du Douro, la limite méridionale de Callaecia et frontière de la province de Lusitania. Voici l'espace et le temps dont nous devons situer le contexte de la construction des mosaïques polychromes identifiées dans le sous-sol de l'église de Santa Maria do Freixo.

Comme dans d'autres centres urbains, dans cette région et au-delà, est une période de profondes transformations. La vitalité de la vie urbaine semble rester uniquement dans les villes qui sont promus à siège du diocèse et, en autre échelle, dans les centres urbains secondaires qui seraient transformés en extensions ruraux du pouvoir épiscopalien, plus tard appelé sièges des «paroisses suèves ». Dans ces dernières, comme à Tongobriga, la construction d'un grand bâtiment revêtu de mosaïque est en contradiction avec la tendance pour la plupart de ces transformations, indicateurs d'un manque de qualité constructive, perte de vitalité économique et désinvestissement dans les bâtiments publics.

Est, en revanche, une époque dont la vallée du Douro, Douro navigable en particulier, est très loin de s'avoir transformé, à la fin de l'Empire, dans une région isolée et économiquement déprimée. Capable, d'après Strabon, d'être navigué par grands navires sur une distance de quasi 800 «stades» (Geographica, 3.3.4.) et considéré par Pline-le-Vieux comme «l'un des grands fleuves d'Hispania» (Plin.nat. 4.112 apud Guerra 1995), le fleuve Douro était une voie de pénétration à partir de l'Océan Atlantique et aussi un canal de drainage des produits de l'hinterland et de contacts commerciaux et aussi culturels.
Figure 19

Localisation des mosaïques connues dans le cours terminal du Douro (Douro navigable) avec représentation schématique du tracé des principales voies romaines (Bracara Augusta Olisippo, Bracara Augusta - Emerita Augusta et Aquae Flaviae - Vissaium)

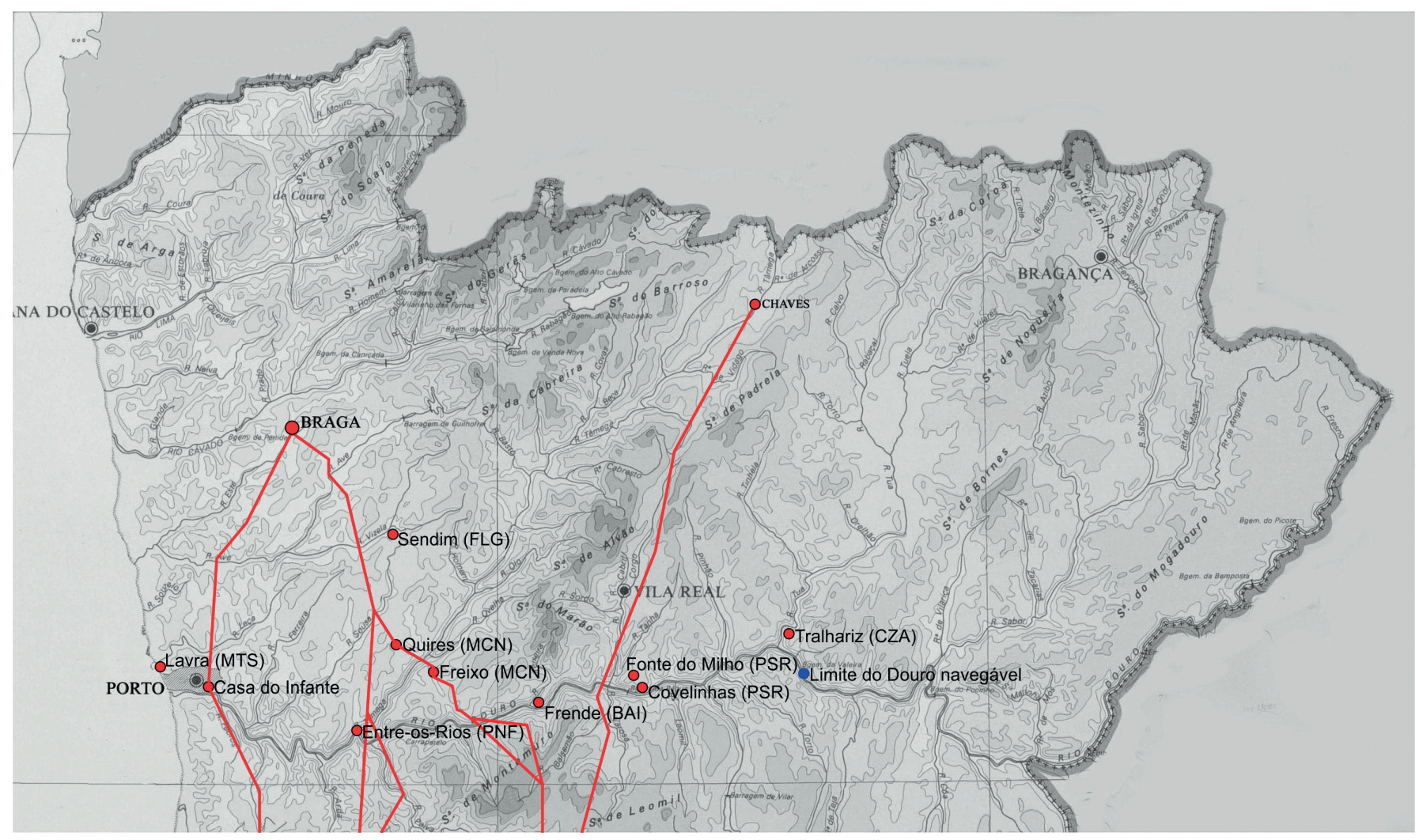


C'est ce contexte géographique qui permet la compréhension des plusieurs vecteurs qui caractérisent le parcours terminal du Douro dans l'Antiquité Tardive, tant sur le plan de la présence de matériaux importés, tant en ce qui concerne les différentes manifestations artistiques qui indiquent une grande ouverture sociale, économique et culturel - à l'Atlantique, aussi à l'Afrique du Nord et à la Méditerranée Orientale, ouverture qui a été sans doute stimulée par un commerce fluvial florissant, particulièrement active à partir du Vème siècle.

Est le moment où se produisent, dans cette région, les premiers indices, souvent indirects, de la diffusion du christianisme dans les zones rurales. C'est aussi l'époque où les enterrements envahissent l'espace d'habitation, bien qu'il soit encore nécessaire de préciser comment les deux phénomènes s'imbriquent.

Contrairement à ce qui semble réussir dans le Haut-Douro (Cortez 1946) au-delà de la montagne du Marão et du Douro navigable, conformément aux différents matrices de peuplement, les mosaïques du cours terminal de ce fleuve sont principalement associées avec des noyaux de peuplement urbain (vici et civitates), quelques d'entre eux étaient lieux saillant durant la période du royaume suève (Almeida 1986: 19; Fernandes 1997: 71), possèdent une chronologie généralement tardive (Almeida 1975 : 38; Maciel 1996: 164-166) et présentent des motifs décoratifs de nature géométrique et/ou végétal (Fig. 19).

Sera l'intégration des mosaïques de Tongobriga dans ce contexte économique et social et dans l'ensemble des manifestations tardives de survivance des élites hispano-romaines en des agglomérations urbaines secondaires du Vème siècle qu'on doit chercher la compréhension de leur signification.

\section{Bibliography - Kaynaklar}

Almeida 1975

Almeida 1986

Blanchard et al. 1973

Caetano 2008

Cortez 1946

David 1947

Décor I

Dias 1997

Fernandes 1997

Fontes 2009

Guerra 1995

Lancha - André 2000

Lima 2003
C. A. F. de Almeida, "Sondagens Arqueológicas em Frende (Baião)", Archaeologica Opuscula. Miscelânea de Arqueologia Nortenha, 1, 29-40.

C. A. F. de Almeida, Arte da Alta Idade Média, História da Arte em Portugal, 2, Lisboa.

M. Blanchard et al., "Répertoire Graphique du Décor Géométrique dans le Mosaïque Romaine", Bulletin de l'Association Internationale pour l'Etude de la Mosaïque Antique, 4, Paris.

M. T. Caetano, "Mosaicos da Villa Romana de São Miguel de Odrinhas. Contributos para uma Nova Leitura", Revista de História de Arte 6, 43-59.

F. R. Cortez, "Os Mosaicos Romanos do Douro", Anais do Instituto do Vinho do Porto 7, 121-161.

P. David, Études Historiques sur la Galice et le Portugal du VI ${ }^{\mathrm{e}}$ au XII ${ }^{\mathrm{e}}$ Siècle, Lisboa-Paris, Liv. Portugália / Les Belles Lettres.

C. Balmelle - M. Blanchard Lemée - J. Christophe - J. P. Darmon - A. M. Guimier Sorbets - H. Lavagne R. Prudhomme - H. Stern, Le Décor Géométrique de la Mosaïque Romaine I, Paris, Picard, 1985.

L. T. Dias, Tongobriga, Lisboa, IPPAR.

A. de A. Fernandes, Paróquias Suevas e Dioceses Visigóticas, Arouca, Associação para a Defesa da Cultura Arouquense / Câmara Municipal de Arouca.

L. Fontes, "A Igreja Sueva de São Martinho de Dume. Arquitectura Cristã Antiga de Braga e na Antiguidade Tardia do Noroeste de Portugal”, Revista de História de Arte 7, 180-203.

A. M. R. Guerra, Plínio-O-Velho e a Lusitânia, Lisboa, Colibri.

J. Lancha - P. André, Corpus dos Mosaicos Romanos de Portugal: II. Conventus Pacensis. I. A Villa de Torre de Palma, Lisboa, IPM / Missão Luso-Francesa "Mosaicos do Sul de Portugal".

A. M. Lima, Área Arqueológica de Freixo. Tongobriga. Trabalhos Arqueológicos 2001 - 2003, Relatório Síntese, IPPAR. 
Lima 2012

Lima 2016a

Lima 2016b

López Monteagudo 2002

Maciel 1996

Munsell 1992

Oliveira 2003

Oliveira 2010

Regueras Grande 2013

Rocha et al. 2015
A. M. Lima, Os mosaicos da ecclesia de Tongobriga, paróquia da diocese portucalense no século VI, Cadernos de Tongobriga 1, Porto.

A. M. Lima, Mudar de Vida. Catálogo da Exposição Permanente do Centro Interpretativo de Tongobriga, Porto.

A. M. Lima, "Os mosaicos da ecclesia de Tongobriga, paróquia da diocese portucalense no século VI", M. J. Maciel - C. Mourão (eds.), Imagens do Paradeisos nos Mosaicos da Hispania, col. Classical and Byzantine Monographs LXXXV, Amsterdam, Adolf M. Hakkert Publisher.

G. López Monteagudo, “Mosaicos Romanos y Elites Locales en el N. de África y en Hispânia”, AEspA 75, 251-268.

M. J. Maciel, Antiguidade Tardia e Paleocristianismo em Portugal, Lisboa, Colibri.

Munsell Soil Color Charts, New York, MacBeth Division of Kollmorgan Instruments.

C. F. de Oliveira, A Villa Romana de Rio Maior. Estudo de Mosaicos, Trabalhos de Arqueologia, 31, Lisboa.

C. F. de Oliveira, Mosaicos Romanos de Portugal. O Algarve Oriental, Tese de Doutoramento, Universidade de Coimbra, Coimbra.

F. Regueras Grande, "Villaquejida, veinte años después: entre la nostalgia y el remordimiento", Brigecio 23, 297-302.

C. Rocha - L. T. Dias - P. Alarcão, Tongobriga. Reflexões sobre o seu desenho urbano, CITCEM / Afrontamento, Porto. 